\title{
openheart Real-world treatment switching to sacubitril/valsartan in patients with heart failure with reduced ejection fraction: A cohort study
}

\author{
Sashiananthan Ganesananthan (1) , ${ }^{1,2}$ Nisar Shah, ${ }^{2}$ Parin Shah, ${ }^{2}$ Hossam Elsayed, ${ }^{2}$ \\ Julie Phillips, ${ }^{2}$ Ann Parkes, ${ }^{2}$ Angharad Morgan, ${ }^{3}$ Zaheer Yousef ${ }^{2}$
}

\begin{abstract}
- Additional material is published online only. To view, please visit the journal online (http://dx.doi.org/10.1136/ openhrt-2020-001305).
\end{abstract}

To cite: Ganesananthan S, Shah N, Shah P, et al. Realworld treatment switching to sacubitril/valsartan in patients with heart failure with reduced ejection fraction: A cohort study. Open Heart 2020;7: 001305. doi:10.1136/

openhrt-2020-001305

SG and NS are joint first authors.

Received 4 April 2020

Revised 14 June 2020

Accepted 2 September 2020
Check for updates

(C) Author(s) (or their employer(s)) 2020. Re-use permitted under CC BY-NC. No commercial re-use. See rights and permissions. Published by BMJ.

${ }^{1}$ School of Medicine, Cardiff University, Cardiff, UK

2Department of Cardiology, University Hospital of Wales,

Cardiff, UK

${ }^{3}$ Health Economics and Outcomes Research Ltd, Cardiff University, Cardiff, UK

Correspondence to Sashiananthan Ganesananthan; GanesananthanS@cardiff.ac.uk

\section{ABSTRACT}

Background Sacubitril/valsartan is an effective treatment for heart failure with reduced ejection fraction (HFrEF) based on clinical trial data. However, little is known about its use or impact in real-world practice. The aim of this study was to describe our routine clinical experience of switching otherwise optimally treated patients with HFrEF to sacubitri//valsartan with respect to patient outcomes such as quality of life (QoL) and echocardiographic variables.

Methods and results From June 2017 to May 2019, 80 consecutive stable patients with HFrEF on established and maximally tolerated guideline-directed $\mathrm{HF}$ therapies were initiated on sacubitril/valsartan with bimonthly uptitration. Clinical assessment, biochemistry, echocardiography and QoL were compared pretreatment and post-treatment switching. We were able to successfully switch $89 \%$ of patients from renin-angiotensin axis inhibitors to sacubitril/valsartan (71 of 80 patients). After 3 months of switch therapy, we observed clinically significant and incremental improvements in blood pressure (systolic blood pressure 123 vs $112 \mathrm{~mm} \mathrm{Hg}, p<0.001$; diastolic blood pressure 72 vs $68 \mathrm{~mm} \mathrm{Hg}, p=0.004$ ), New York Heart Association functional classification score (2.3 vs $1.9, p<0.001$ ), Minnesota Living with Heart Failure Questionnaire score (46 vs $38, p=0.016$ ), left ventricular ejection fraction $(26 \%$ vs $33 \%, p<0.001)$ and left ventricular end systolic diameter (5.2 vs $4.9 \mathrm{~cm}, \mathrm{p}=0.013$ ) compared with baseline. There were no significant changes in renal function or serum potassium.

Conclusion This study provides real-world clinical practice data demonstrating incremental improvements in functional and echocardiographic outcomes in optimally treated patients with HFrEF switched to sacubitril/valsartan. The data provide evidence beyond that observed in clinical trial settings of the potential benefits of sacubitril/valsartan when used as part of a multidisciplinary heart failure programme.

\section{INTRODUCTION}

The clinical and economic burden of heart failure $(\mathrm{HF})$ is significant; 5-year mortality is $45 \%-60 \%^{1}$; and with approximately half a million patients with $\mathrm{HF}$ in the $\mathrm{UK}^{2}{ }^{2}$ it is

\section{Key questions}

What is already known about this subject?

- The randomised, double-blind Prospective Comparison of Angiotensin-Neprilysin Inhibition with ACEI to Determine Impact on Global Mortality and Morbidity trial in patients with heart failure with reduced ejection fraction (HFrEF) established a $20 \%$ relative reduction of the primary endpoint (composite of cardiovascular death or heart failure hospitalisation) and a $16 \%$ relative risk reduction of all-cause mortality.

What does this study add?

- This study provides real-world functional and echocardiographic data of the incremental benefit of sacubitril/valsartan in patients with stable HFrEF who were already on optimal medical therapy. We have demonstrated that switching patients from ACE inhibitor/angiotensin receptor blocker to sacubitril/valsartan is tolerable, safe and leads to significant improvement in left ventricular function and quality of life.

How might this impact on clinical practice?

- Our data show a real-world incremental benefit and safety of sacubitril/valsartan in patients with HFrEF who were already on evidence-based optimal medical treatment. Sacubitril/valsartan should be part of routine clinical practice for patients with HFrEF.

estimated that $1 \%-2 \%$ of the NHS budget is spent on $\mathrm{HF}$, with $60 \%-70 \%$ related to the costs of hospitalisation. ${ }^{3}$ The early diagnosis and treatment of $\mathrm{HF}$ are essential to improving patients' quality and quantity of life. Until recently, the mainstay of pharmacological treatment for patients with heart failure with reduced ejection fraction (HFrEF) has included ACE inhibitors (ACE-i's) or angiotensin receptor blockers (ARBs) and beta blockers as first-line therapy, and mineralocorticoid receptor antagonists (MRAs) as second-line therapy. ${ }^{4}$ More 
recently, the European Medicine Agency, US Food and Drug Administration and UK National Institute for Health and Clinical Excellence have approved sacubitril/valsartan, a combined neprilysin inhibitor (sacubitril) and an ARB (valsartan), which may be prescribed as an alternative to ACE-i's or ARBs for the treatment of HFrEF. ${ }^{5-7}$ Following the approvals, there have been strong class I recommendation in both US and European Union HF clinical guidelines. Guidelines from the European Society of Cardiology recommend switching to sacubitril/valsartan in patients who remain symptomatic despite optimal treatment with an ACE-i/ARB, a betablocker or an MRA. ${ }^{8}$

These recommendations are based on compelling evidence from the Prospective Comparison of Angiotensin-Neprilysin Inhibition with ACEI to Determine Impact on Global Mortality and Morbidity in Heart Failure (PARADIGM-HF) study, a randomised, doubleblind and event-driven trial which compared sacubitril/ valsartan with enalapril in 8442 patients with HFrEF. ${ }^{9}$ Sacubitril/valsartan demonstrated superiority with a $20 \%$ reduction in the primary endpoint, a composite of death from cardiovascular causes or hospitalisation for HF. Sacubitril/valsartan also had a lower rate $(16 \%$ relative risk reduction) of all-cause mortality.

Real-world data are important to understand the generalisability and applicability of sacubitril/valsartan in realworld clinical practice and to help inform everyday patient treatment decisions for clinicians managing patients with HF. To date, there are limited real-world data for sacubitril/valsartan in HF. We previously assessed the eligibility of patients in a secondary care HF service for treatment with sacubitril/valsartan and found that that 31\%-37\% of patients with HFrEF were established on maximally tolerated guideline-directed ACE-i or ARB and may be suitable for treatment with sacubitril/valsartan. ${ }^{10}$ Initial clinical experience of sacubitril/valsartan in UK HF services have also shown a favourable safety and tolerability profile. ${ }^{11} 12$ The aim of this study was to describe our real-word experience of switching stable and optimally medicated patients with HFrEF to sacubitril/valsartan with respect to quality of life (QoL) and echocardiographic outcomes, in addition to safety and tolerability outcomes.

\section{METHODS}

\section{Study population}

This is a retrospective cohort of 80 consecutive patients with a diagnosis of stable HFrEF (defined as symptoms and/or signs of HF, New York Heart Association (NYHA) Functional Classification of $\geq \mathrm{II}$ and a reduced left ventricular ejection fraction (LVEF) of $\leq 35 \%$ measured by echocardiography) attending a nurse-led sacubitril/valsartan titration clinic within Cardiff and Vale University Health Board (University Hospital of Wales) from June 2017 to May 2019. Patients included were sequentially referred to this HF service via an open access referral system from secondary care physicians, general practitioners and cardiologists, including referrals from the tertiary HF service from our institution. We include the protocol of this nurse-led service in the online supplementary material.

Patient medications had already been aggressively optimised as previously described ${ }^{13}$ and were deemed to be on optimal evidence-based medical therapy. ${ }^{8}$ Patients had been assessed as potentially suitable for treatment with sacubitril/valsartan ${ }^{10}$ and were regularly monitored and discussed at weekly multidisciplinary HF team meetings.

\section{Treatment switching to sacubitril/valsartan}

Following a 48-hour ACE-i washout period (due to the higher risk of angioedema if these drugs are combined), patients were initiated on sacubitril/valsartan at a dose according to the dose of ACE-i or ARB. Clinic visits to optimise treatment were scheduled bimonthly with slow uptitration of sacubitril/valsartan, with careful monitoring of side effects, heart rate, blood pressure and biochemistry. Titration continued until the patient either was taking the maximum recommended dose or reported side effects. Other evidence/guideline-based medications were continued where possible but, in some cases, were reduced to initiate sacubitril/valsartan.

\section{Study outcomes}

Baseline evaluation included clinical assessment, biochemistry, echocardiography and QoL score using the Minnesota Living with Heart Failure Questionnaire (MLHFQ). ${ }^{14}$ Three months after uptitration of sacubitril/valsartan to its maximally tolerated dose, patient outcomes were re-evaluated.

\section{Statistical analysis}

Categorical data are presented as numbers and percentages and continuous data as mean \pm SD. Differences in baseline and postoptimisation patient outcomes were analysed using paired sample t-tests. All statistical analyses were carried out using SPSS V.25.0.0.1, and $\mathrm{p}<0.05$ was used to indicate statistical significance.

\section{Patient and public involvement}

There was no patient or public involvement in the generation of our research article. Patients or the public were not involved in the creation of the research question, study execution, interpretation or dissemination of these results.

\section{RESULTS \\ Baseline patient characteristics}

Baseline characteristics of the 80 patients included in this study are presented in table 1 . The mean age was $64 \pm 12$ years and $73 \%$ of patients were male. With regard to NYHA functional classification, $74 \%$ of the patients were class II; $22 \%$ were class III; and $4 \%$ were class IV. At baseline, all patients were receiving either an ACE-i (74\%) or 


\begin{tabular}{|c|c|}
\hline \multicolumn{2}{|l|}{ Baseline patient characteristics } \\
\hline Age (years) & $64 \pm 12$ \\
\hline Gender (male) & $58(73)$ \\
\hline Systolic blood pressure (mm Hg) & $122 \pm 18$ \\
\hline Heart rate (beats/min) & $71 \pm 11$ \\
\hline Weight (kg) & $90 \pm 22$ \\
\hline Estimated glomerular filtration rate $\left(\mathrm{mL} / \mathrm{min} / 1.73 \mathrm{~m}^{2}\right)$ & $68 \pm 17$ \\
\hline Serum creatinine (mmol/L) & $97 \pm 27$ \\
\hline Serum potassium (mmol/L) & $4.6 \pm 0.4$ \\
\hline Ischaemic cardiomyopathy & $38(48)$ \\
\hline Left ventricular ejection fraction (\%) & $26 \pm 7$ \\
\hline Left ventricular end systolic diameter (cm) & $5 \pm 1$ \\
\hline Left ventricular end diastolic diameter (cm) & $6 \pm 1$ \\
\hline NYHA I & $0(0)$ \\
\hline NYHA II & $59(74)$ \\
\hline NYHA III & $18(22)$ \\
\hline NYHA IV & $3(4)$ \\
\hline $\begin{array}{l}\text { Mean Minnesota Living with Heart Failure } \\
\text { Questionnaire }\end{array}$ & $45(26)$ \\
\hline Hypertension & $26(33)$ \\
\hline Diabetes & $17(21)$ \\
\hline Atrial fibrillation & $28(35)$ \\
\hline Cerebrovascular accident & $9(11)$ \\
\hline ACE inhibitor & $61(76)$ \\
\hline Angiotensin receptor blocker & $19(24)$ \\
\hline Beta blocker & $76(95)$ \\
\hline Mineralocorticoid receptor antagonist & $73(91)$ \\
\hline Loop diuretics & $42(53)$ \\
\hline Digitalis & $17(21)$ \\
\hline Nitrates & $9(11)$ \\
\hline Aspirin & $23(29)$ \\
\hline Clopidogrel/prasugrel/ticagrelor & $5(6)$ \\
\hline Warfarin & $20(21)$ \\
\hline Direct oral anticoagulant & $16(20)$ \\
\hline Statin & $55(69)$ \\
\hline CRT P & $4(5)$ \\
\hline CRT D & $27(34)$ \\
\hline Implantable cardioverter defibrillator & $10(13)$ \\
\hline
\end{tabular}

Categorical variables are expressed as number (percentage) and continuous variables are expressed as mean (SD) or median (IQR) depending on distribution.

CRT, cardiac resynchronisation therapy; NYHA, New York Heart Association.

ARB (24\%); $95 \%$ were on a beta blocker; and $91 \%$ were taking an MRA.

\section{Treatment switching}

Of the 80 patients included in this study, 7 could not tolerate sacubitril/valsartan (severe symptomatic

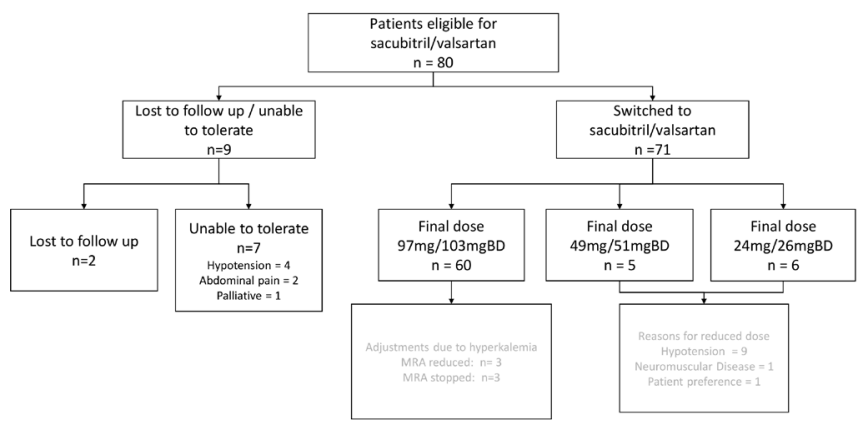

Figure 1 Dose optimisation and side effects of treatment switching in the patients enrolled in our study. MRA

hypotension: $\mathrm{n}=4$, severe abdominal pain, nausea and vomiting: $\mathrm{n}=2$ and palliative patient $\mathrm{n}=1$ ) and 2 were lost to follow-up. Of the 71 patients titrated to a stable final dose of sacubitril/valsartan, 60 reached the maximum recommended dose of $97 \mathrm{mg} / 103 \mathrm{mgBD}$. However, as a consequence of hyperkalaemia, $5 \%$ of these patients had their MRA dose reduced, and a further $5 \%$ had their MRA discontinued in order to achieve the maximum dose of sacubitril/valsartan. Eleven patients did not reach the maximum dose of sacubitril/valsartan due to hypotension $(n=9)$, neuromuscular disease $(n=1)$ or patient preference $(n=1)$. Five of these patients were on an intermediate final dose of $49 \mathrm{mg} / 51 \mathrm{mgBD}$, and six patients were on a low final dose of $24 \mathrm{mg} / 26 \mathrm{mgBD}$ (figure 1).

The mean time from initiation of sacubitril/valsartan to the final stable dose was $117 \pm 9$ days.

\section{Changes in patient outcomes following treatment switching to sacubitril/valsartan}

Comparing pretreatment and post-treatment switching to sacubitril/valsartan, we found that there were statistically significant improvements in blood pressure (systolic blood pressure 123 vs $112 \mathrm{~mm} \mathrm{Hg}, \mathrm{p}<0.001$; diastolic blood pressure 72 vs $68 \mathrm{~mm} \mathrm{Hg}, \mathrm{p}=0.004$ ), NYHA score $(2.3$ vs $1.9, \mathrm{p}<0.001)$ and MLHFQ score (46 vs 38 , $\mathrm{p}=0.016)$ (table 2).

With regard to echocardiographic measures, there was a significant increase in LVEF (26 vs 33\%, p<0.001) and significant decreases in left ventricular end systolic diameter (LVESD) ( $5.2 \mathrm{v} 4.9 \mathrm{~cm}, \mathrm{p}=0.013)$ and left ventricular end diastolic diameter (LVEDD) (6.0 vs $5.7 \mathrm{~cm}, \mathrm{p}=0.042$ ) postoptimisation with sacubitril/valsartan compared with baseline (table 2).

There were no significant changes in renal function (creatinine or estimated glomerular filtration rate) or serum potassium (allowing for changes in MRA dosing) following treatment switching to sacubitril/valsartan (table 2).

\section{DISCUSSION}

Our real-world experience suggests that switching treatment to sacubitril/valsartan in otherwise stable patients 


\begin{tabular}{|c|c|c|c|c|c|c|}
\hline & $\mathbf{n}$ & Pre-switching & Post-switching & Mean difference (SD) & $95 \% \mathrm{Cl}$ & $P$ value \\
\hline $\mathrm{SBP}(\mathrm{mm} \mathrm{Hg})$ & 68 & 123 & 112 & $-10(14)$ & -14 to -7 & $<0.001$ \\
\hline $\mathrm{DBP}(\mathrm{mm} \mathrm{Hg})$ & 68 & 72 & 68 & $-4(10)$ & -6 to -1 & 0.004 \\
\hline NYHA score & 71 & 2.3 & 1.9 & $-0.4(0.63)$ & -0.6 to -0.2 & $<0.001$ \\
\hline MLHFQ score & 33 & 46 & 38 & $-9(19)$ & -15 to -2 & 0.016 \\
\hline $\operatorname{LVEF}(\%)$ & 49 & 26 & 33 & $7(10)$ & 4 to 10 & $<0.001$ \\
\hline LVESD (cm) & 37 & 5.2 & 4.9 & $-0.3(0.8)$ & -0.6 to -0.08 & 0.013 \\
\hline LVEDD (cm) & 48 & 6.0 & 5.7 & $-0.3(0.7)$ & -0.5 to -0.1 & 0.042 \\
\hline $\mathrm{K}^{+}(\mathrm{mmol} / \mathrm{L})$ & 71 & 4.6 & 4.7 & $0.1(0.40)$ & -0.01 to 0.20 & 0.054 \\
\hline Creatinine $(\mu \mathrm{mol} / \mathrm{L})$ & 71 & 95 & 97 & $2(14)$ & -1 to 6 & 0.17 \\
\hline eGFR $\left(\mathrm{mL} / \mathrm{min} / 1.73 \mathrm{~m}^{2}\right)$ & 71 & 69 & 67 & $-2(13)$ & -5 to 1 & 0.23 \\
\hline
\end{tabular}

DBP, diastolic blood pressure; eGFR, estimated glomerular filtration rate; $\mathrm{K}^{+}$, potassium; LVEDD, left ventricular end diastolic diameter;

LVEF, left ventricular ejection fraction; LVESD, left ventricular end systolic diameter; MLHFQ, Minnesota Living with Heart Failure

Questionnaire; NYHA, New York Heart Association; SBP, systolic blood pressure.

with HFrEF established on optimal guideline-directed medical therapy results in significant improvements of cardiac structure and function, QoL, HF symptoms as assessed by the physician and functional capacity, with no significant change in renal function.

There are a limited number of studies on the real-world experience of switching to sacubitril/valsartan in patients with HFrEF. A single-centre, retrospective, cohort study of 48 patients with HFrEF who were treated with sacubitril/valsartan for a median duration of 3 months demonstrated improvements in EF and multiple measures of reverse remodelling, including reduced LVESD, LVEDD and left ventricular mass with sacubitril/valsartan. ${ }^{15}$ Another study examined data from medical and pharmacy claims and medical records of 200 patients with HFrEF and found symptom improvements (fatigue and shortness of breath) and a reduction in hospitalisations within 4 months of sacubitril/valsartan treatment. ${ }^{16}$ Our study, however, is the first to describe a range of both functional and echocardiography outcomes after switching to sacubitril/valsartan in a multidisciplinary nurse-led HF service. These real-world data provide mechanistic insights and support the overwhelming survival and HF hospitalisation benefits demonstrated in the PARADIGM study. ${ }^{17} 18$

Our data are in concordance with secondary analysis from the PARADIGM trial, describing significant improvements in the Kansas City Cardiomyopathy Questionnaire (KCCQ) clinical and overall summary scores, ${ }^{17}$ similar to the effect size in health-related QoL levels with cardiac resynchronisation therapy observed in the MADIT-CRTstudy (Multicenter Automatic Defibrillator Implantation Trial with Cardiac Resynchronization Therapy in HFrEF. ${ }^{19}$ Focusing on individual physical and social activities items in the KCCQ, a subsequent analysis provided further granularity of the effects of sacubitril/ valsartan, with largest responses in household chores and sexual relationships. ${ }^{20}$
Sacubitril/valsartan is tolerated well, and studies have shown that even earlier initiation is still tolerable. The TITRATION study (Safety and Tolerability of Initiating LCZ696 in Heart Failure Patients), which was a randomised comparison of two uptitration regimens (a 'condensed' 3-week regimen or 'conservative' 6-week regimen), showed a similar tolerability profile between the two regimens and was in line with other HF treatments such as ACE-i/ARBs. ${ }^{21}$ The TRANSITION study (Comparison Pre- and Post-discharge Initiation of LCZ696 Therapy in HFrEF Patients After an Acute Decompensation Event) ${ }^{22}$ provided complementary data to the PIONEER-HF study (Comparison of Sacubitril-Valsartan versus Enalapril on Effect on NT-proBNP in Patients Stabilized from an Acute Heart Failure Episode), ${ }^{23}$ further suggesting that early initiation and uptitration of sacubitril/valsartan were generally successful even in high-risk but stabilised patients, following acute decompensated HF.

The most common symptomatic adverse event associated with sacubitril/valsartan in our study, as well as in PARADIGM-HF trial, was hypotension. In our study, 15\% of patients who were successfully switched to sacubitril/ valsartan received a lower than recommended dose, mainly due to hypotension. Furthermore, hypotension meant that some patients could not tolerate sacubitril/ valsartan at all and hence failed the uptitration period.

\section{Limitations}

Limitations of this real-world study include the relatively small sample of sequential patients with short follow-up and the inherent bias of patients not referred into the HF clinic. Although our follow-up drop-out rate of $8.75 \%$ (seven patients) further diluted our dataset, we believe this is reflective of real-world practice and in keeping with the drop-out rate $(9.4 \%)$ experienced in the PARADIGM clinical trial. $^{9}$

The relatively young age group (mean age of 64 years) in our study and previous studies ${ }^{912}$ may preclude 
extrapolation to an older cohort. Older patients are more likely to have more advanced disease with a range of comorbidities, such as renal impairment or autonomic dysfunction. However, real-world studies such as ours aim to provide objective evidence and increase familiarity with sacubitril/valsartan to inform future use in this patient population. Furthermore, a lack of a comparator group may not allow a direct comparison of patients switching to sacubitril/valsartan to those who remain on an ACE-i or ARB. However, as all patients were already treatment optimised prior to switching to sacubitril/valsartan, each patient was effectively acting as his or her own control. Hence, any changes observed in patient outcomes are likely attributable to sacubitril/valsartan. Serial natriuretic peptide levels to guide optimisation of medical therapy in HF were not measured during the course of this study. ${ }^{24}$

Although optimal sacubitril/valsartan therapy may be limited in a small number of patients due to symptomatic hypotension, our real-world experience of treatment switching to sacubitril/valsartan in patients with HFrEF demonstrates significant improvements in patient outcomes for the majority of patients with HFrEF who are able to tolerate this treatment switching. These encouraging results require confirmation in larger prospective cohorts with longer follow-up.

\section{CONCLUSION}

Sacubitril/valsartan have already proven to be beneficial for patients with HFrEF in clinical trial data. The data from this study provide real-world evidence that corroborates the benefits of sacubitril/valsartan in real-life clinical practice and suggest that sacubitril/valsartan should be routinely considered by clinicians as part of optimal medical treatment for patients with HFrEF.

Contributors Study design and conception: SG, NS, PS and ZY. Data acquisition: SG and NS. Manuscript drafting: SG, PS, AM and ZY. Data analysis and interpretation and critical revision of the manuscript: all authors.

Funding The authors have not declared a specific grant for this research from any funding agency in the public, commercial or not-for-profit sectors.

Competing interests $Z Y$ has received lecture fees and research funding from Novartis.

Patient consent for publication Not required.

Ethics approval The main aim of the study was to describe the real-world experience of switching patients from ACE inhibitor/angiotensin receptor blockers to angiotensin receptor neprilysin inhibitor (ARNI). Hence, formal ethical approval was not required for this non-randomised and observational study as it was conducted as part of a service evaluation project at Cardiff \& Vale Health Trust. Guidance advocated by the World Medical Association's Declaration of Helsinki was complied with throughout. The patient data were anonymised for data collection, outcome analysis and publication.

Provenance and peer review Not commissioned; externally peer reviewed.

Data availability statement Data are available upon reasonable request.

Open access This is an open access article distributed in accordance with the Creative Commons Attribution Non Commercial (CC BY-NC 4.0) license, which permits others to distribute, remix, adapt, build upon this work non-commercially, and license their derivative works on different terms, provided the original work is properly cited, appropriate credit is given, any changes made indicated, and the use is non-commercial. See: http://creativecommons.org/licenses/by-nc/4.0/.
ORCID iD

Sashiananthan Ganesananthan http://orcid.org/0000-0001-7503-7527

\section{REFERENCES}

1 Levy D, Kenchaiah S, Larson MG, et al. Long-term trends in the incidence of and survival with heart failure. $N$ Engl J Med 2002;347:1397-402.

2 Cowie MR. The heart failure epidemic: a UK perspective. Echo Res Pract 2017;4:R15-20.

3 Braunschweig F, Cowie MR, Auricchio A. What are the costs of heart failure? Europace 2011;13:ii13-17.

4 McMurray JJV, Adamopoulos S, Anker SD, et al. ESC guidelines for the diagnosis and treatment of acute and chronic heart failure 2012: the task force for the diagnosis and treatment of acute and chronic heart failure 2012 of the European Society of cardiology. developed in collaboration with the heart failure association (HFA) of the ESC. Eur J Heart Fail 2012;14:803-69.

5 US Food and Drug Administration. ENTRESTO (sacubitril and valsartan). highlights of prescribing information, 2019. Available: http://www.accessdata.fda.gov/drugsatfda_docs/label/2015/ 2076200rig1s000lbl.pdf

6 European Medicines Agency (EMA). Summary of the risk management plan (RMP) for Entresto (sacubitril/valsartan), 2019. Available: http://www.ema.europa.eu/documents/rmp-summary/ entresto-epar-risk-management-plan-summary_en.pdf

7 National Institute for Health and Clinical Excellence (NICE). Technology appraisal guidance [TA388]: sacubitril valsartan for treating symptomatic chronic heart failure with reduced ejection fraction, 2016. Available: https://www.nice.org.uk/guidance/ta388

8 Ponikowski P, Voors AA, Anker SD, et al. 2016 ESC Guidelines for the diagnosis and treatment of acute and chronic heart failure: The Task Force for the diagnosis and treatment of acute and chronic heart failure of the European Society of Cardiology (ESC)Developed with the special contribution of the Heart Failure Association (HFA) of the ESC. Eur Heart J 2016;37:2129-200.

9 McMurray JJV, Packer M, Desai AS, et al. Angiotensin-neprilysin inhibition versus enalapril in heart failure. N Engl J Med 2014;371:993-1004.

10 Lodge FM, Phillips J, Groves T, et al. Eligibility of patients for sacubitril/valsartan in a secondary care heart failure service. $\mathrm{Br} J$ Cardiol 2016;23:148-50.

11 Ali D, Hyland J, Riley F, et al. Early clinical experience with sacubitril/ valsartan from a large UK tertiary centre. Br J Cardiol 2019;26:S9-14.

12 Crawley RJ, Morton G, Kalsi N, et al. Initial experience of introducing sacubitril/valsartan in a UK heart failure service. $\mathrm{Br} J$ Cardiol 2019;26:S15-19.

13 Russell SJ, Oliver M, Edmunds L, et al. Optimised beta blocker therapy in heart failure: is there space for additional heart rate control. Br J Cardiol 2012;19:21-3.

14 Rector TS, Kubo SH, Cohn JN. Patients' self-assessment of their congestive heart failure. Part 2: content, reliability and validity of a new measure, The Minnesota Living with Heart Failure Questionnaire. Heart Fail 1987;3:198-209.

15 Almufleh A, Marbach J, Chih S, et al. Ejection fraction improvement and reverse remodeling achieved with Sacubitril/Valsartan in heart failure with reduced ejection fraction patients. Am J Cardiovasc Dis 2017;7:108-13.

16 Antol DD, Casebeer AW, DeClue RW, et al. An early view of realworld patient response to Sacubitril/Valsartan: a retrospective study of patients with heart failure with reduced ejection fraction. Adv Ther 2018;35:785-95.

17 Lewis EF, Claggett BL, McMurray JJV, et al. Health-related quality of life outcomes in PARADIGM-HF. Circulation 2017;10.

18 Kramer DG, Trikalinos TA, Kent DM, et al. Quantitative evaluation of drug or device effects on ventricular remodeling as predictors of therapeutic effects on mortality in patients with heart failure and reduced ejection fraction: a meta-analytic approach. J Am Coll Cardiol 2010;56:392-406.

19 Moss AJ, Hall WJ, Cannom DS, et al. Cardiac-resynchronization therapy for the prevention of heart-failure events. N Engl J Med 2009;361:1329-38.

20 Chandra A, Lewis EF, Claggett BL, et al. Effects of Sacubitril/ Valsartan on physical and social activity limitations in patients with heart failure: a secondary analysis of the PARADIGM-HF trial. JAMA Cardiol 2018;3:498.

21 Senni M, McMurray JJV, Wachter R, et al. Initiating sacubitril/ valsartan (LCZ696) in heart failure: results of titration, a double-blind, randomized comparison of two uptitration regimens. Eur J Heart Fail 2016;18:1193-202. 
22 Wachter R, Senni M, Belohlavek J, et al. Initiation of sacubitril/ valsartan in haemodynamically stabilised heart failure patients in hospital or early after discharge: primary results of the randomised transition study. Eur J Heart Fail 2019;21:998-1007.
23 Velazquez EJ, Morrow DA, DeVore AD, et al. Angiotensin-neprilysin inhibition in acute decompensated heart failure. $N$ Engl $J$ Med 2019;380:539-48.

24 McLellan J, Heneghan CJ, Perera R, et al. B-type natriuretic peptideguided treatment for heart failure. Cochrane Database Syst Rev 2016;12:CD008966 